\title{
Performance Comparison of WhatsApp versus Skype on Smart Phones
}

\author{
Nayankumar Patel, Swapnil Patel, Wee Lum Tan \\ School of ICT, Griffith University \\ Gold Coast, Australia \\ \{nayankumar.patel, swapnil.patel\}@griffithuni.edu.au,w.tan@griffith.edu.au
}

\begin{abstract}
This paper presents the findings of our study into the performance of two popular instant messaging applications: WhatsApp and Skype. We have evaluated the performance of WhatsApp and Skype in terms of their data consumption/usage when sending text messages and making VoIP calls, as well as the quality of the VoIP calls made with these applications. Our results show that WhatsApp uses less data compared to Skype when sending text messages and making VoIP calls. We also find that both applications have similar VoIP call quality (mean opinion scores) when network conditions are good. However under poor network conditions, WhatsApp VoIP call quality is better than that of Skype. Our results will be useful to smart phone users who have mobile plans with limited data allowance, and provides guidance to them in terms of their selection of which instant messaging app is more suitable for them.
\end{abstract}

Keywords-WhatsApp, Skype, data consumption, VoIP call quality

\section{INTRODUCTION}

Nowadays, instant messaging applications have overtaken the traditional SMS and native voice calling services as the preferred mode of communications on smart phones. WhatsApp and Skype are two of the most popular instant messaging applications available on smart phones. They allow users to send instant text messages, images and documents, and make VoIP and video calls.

WhatsApp and Skype can be used when the smart phones are connected to Wi-Fi networks or cellular networks. In the latter case, a mobile data subscription would be necessary. In some mobile data subscription models, e.g. prepaid with a fixed, limited amount of mobile data allowance per month, the amount of data used by mobile applications can be a critical consideration. In this paper, we evaluate the performance of WhatsApp and Skype in terms of how much data is used when sending text messages and making VoIP calls. We also compare the performance of these two applications in terms of their VoIP call quality parameter, using the Perception Evaluation of Speech Quality (PESQ) testing standard. These performance evaluations are carried out under varying network conditions, characterised by increasing packet loss. Our findings show that WhatsApp uses less data compared to Skype when sending messages and making VoIP calls. In terms of VoIP call quality, we find that both applications have similar Mean Opinion Score (MOS) under good network conditions. However under deteriorating network conditions (packet loss greater than 20\%), WhatsApp's VoIP call quality is better than that of Skype.
There are prior works that have measured and evaluated the performance of VoIP call quality of different VoIP applications including Skype [1], [2], [3], [4], analysis of Skype's traffic and video call quality [5], [6], and forensic analysis of VoIP traffic in different VoIP applications [7], [8], [9]. These works have all concentrated on the VoIP call quality and traffic analysis, in particular with a focus on the Skype messaging application. On the other hand, our work is focused on evaluating the data consumption/usage when sending text messages and making VoIP calls with the WhatsApp and Skype applications.

The rest of this paper is organised as follows. We describe our measurement methodology and testbed configurations in Section II, and present the measurement results in Section III. In Section IV, we conclude the paper and provide some potential future work.

\section{EXPERIMENTAL SETUP}

We have setup an experimental testbed to measure the data consumption performance and VoIP call quality of WhatsApp and Skype, as shown in Fig. 1. In the testbed, we have configured a Windows laptop as a mobile wireless hotspot, providing network connectivity to two smart phones. The laptop itself is connected to the Internet via an ADSL router.

Using this testbed, we run an instance of WhatsApp (or Skype) on smart phone A and another instance of WhatsApp (or Skype) on smart phone B. To measure the data consumption performance of the messaging applications, we run two tests:

- Phone A sends a text message to Phone B

- Phone A makes a VoIP call to Phone B

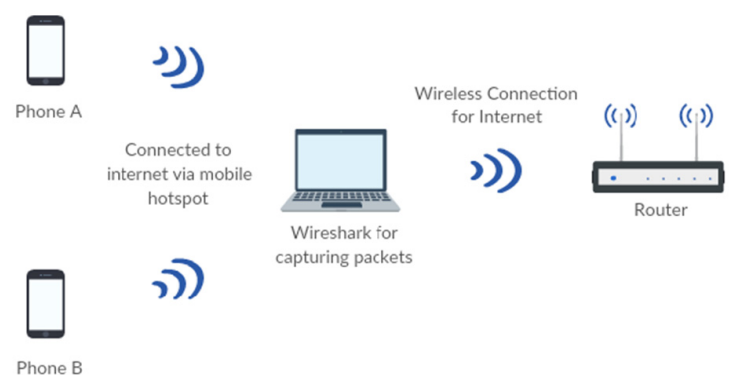

Fig. 1: Experimental testbed 
The text message that we used is a 13-byte string "Hello testing". For the VoIP call, we played a pre-recorded 8seconds audio WAV file that is provided with the PESQ tool, which can be obtained from www.itu.int [12].

In all communications between Phone $\mathrm{A}$ and Phone $\mathrm{B}$, data packets will traverse through the laptop on their way to the WhatsApp/Skype server, and then back through the laptop before arriving at the destination phone. On the laptop, we run Wireshark [10] to capture and filter the data packets corresponding to each phone (Phone A or Phone B) and each messaging application (WhatsApp or Skype). We compute the data consumption parameter by tallying the size of all packets (data and control/signaling packets) that are captured in the Wireshark packet traces.

We also used a network tool on the laptop to emulate packet loss on the network connection between the two phones. The tool we used, Clumsy [11], allows us to set the packet loss rate on the laptop, as shown in Fig. 2. We repeat all our tests for different values of packet loss on the network connection, ranging from $0 \%$ to $50 \%$.

For VoIP call quality measurement, we played the audio WAV file at Phone A and recorded the received audio at Phone B. Using the PESQ tool [12] as shown in Fig. 3, we compute the raw mean opinion score (MOS) for the recording. MOS scores range from one (poor) to five (excellent). We repeat the test for varying packet loss in the network connection, as emulated by the Clumsy tool.

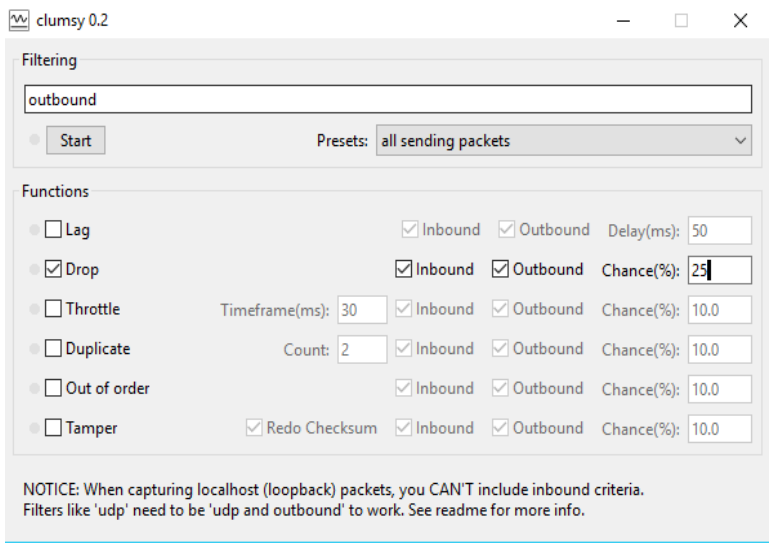

Fig. 2: Clumsy network tool

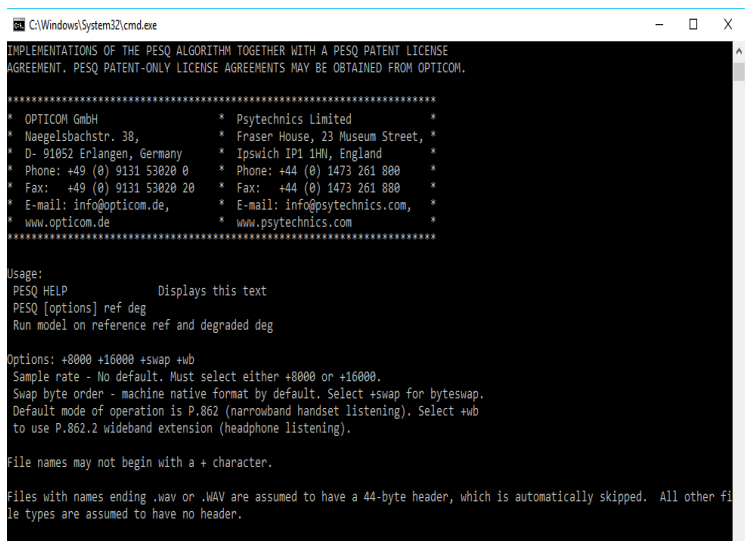

Fig. 3: PESQ tool

\section{RESULTS AND DISCUSSION}

In this section, we present the results from our experiments with the data consumption and VoIP call quality of WhatsApp versus Skype. Each result in the graphs is the computed average of ten separate tests.

\section{A. Data Consumption}

Fig. 4 shows the data consumption of the two messaging applications with increasing packet loss, for the case where a text message was sent from Phone A to Phone $\mathrm{B}$. The data consumption for sending a text message is the total number of bytes in the data and control packets corresponding to the text message session. As we can see in Fig. 4, WhatsApp uses significantly less data compared to Skype when sending and receiving a text message. Since both messaging applications were sending the same text message in the test, this indicates that Skype has more control messaging overhead compared to WhatsApp. We also see in Fig. 4 that increasing packet loss has minimal impact on the data consumption parameter. This could be due to the fact that the text message we used in the test was fairly small in size. In addition, we see that the data consumption in receiving a text message is higher than that of sending a text message. This could be because the WhatsApp/Skype server needs to send additional "wakeup" control packets to an idle/sleeping receiver, before sending the actual text message.

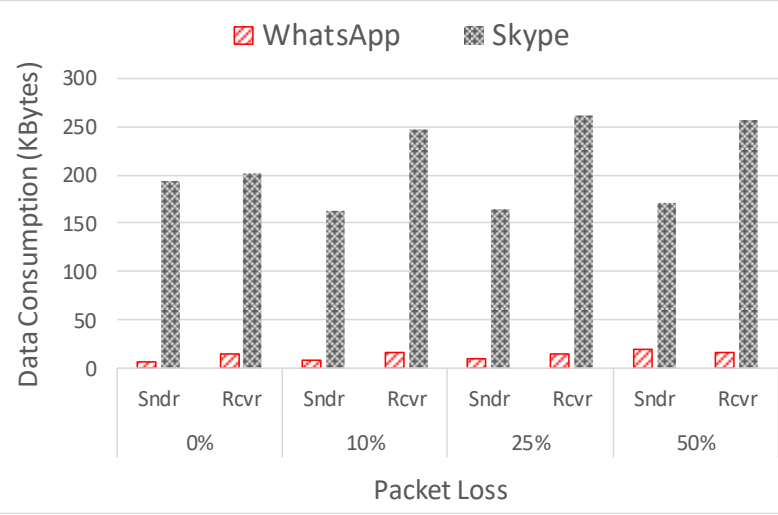

Fig. 4: Data consumption of text message vs packet loss

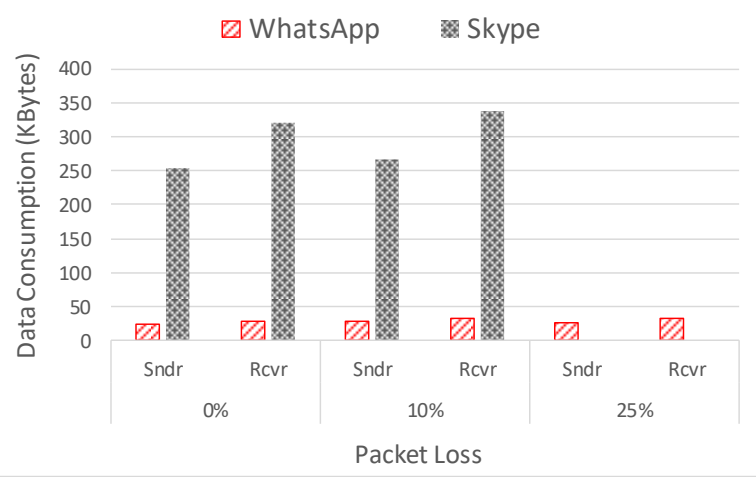

Fig. 5: Data consumption of VoIP call vs packet loss 
Fig. 5 shows the data consumption for making a VoIP call from Phone A to Phone B, using WhatsApp and Skype. In this experiment, we played the audio WAV file at Phone $\mathrm{A}$ and recorded the received audio at Phone B. Both phones are located in different closed rooms, to minimize the amount of background noise during the test. Similar to the results in the test on data consumption for text message, WhatsApp uses significantly less data compared to Skype when making and receiving a VoIP call. In addition, Skype is unable to make a VoIP call when the packet loss rate is $25 \%$ and higher, in contrast to WhatsApp that is still able to make a voice call with a packet loss rate of $25 \%$. This indicates that WhatsApp is more resilient to packet losses compared to Skype.

\section{B. VoIP Call Quality}

Using the PESQ tool [12] as shown in Fig. 3, we compute the raw mean opinion score (MOS) for the VoIP call recording, by comparing it with the original audio WAV file. As mentioned previously, MOS scores range from one (poor) to five (excellent). Fig. 6 shows that when network conditions are good (packet loss $<20 \%$ ), the MOS scores of both WhatsApp and Skype voice calls are pretty similar. However under poor network conditions (packet losses $>=20 \%$ ), it is clear that the quality of a WhatsApp voice call is better than that of a Skype voice call as indicated by the MOS scores in Fig. 6. In addition, we can conclude that compared to Skype, WhatApp is more resilient to poor network conditions as WhatsApp is still able to successfully make a voice call even when packet losses are higher than $25 \%$.

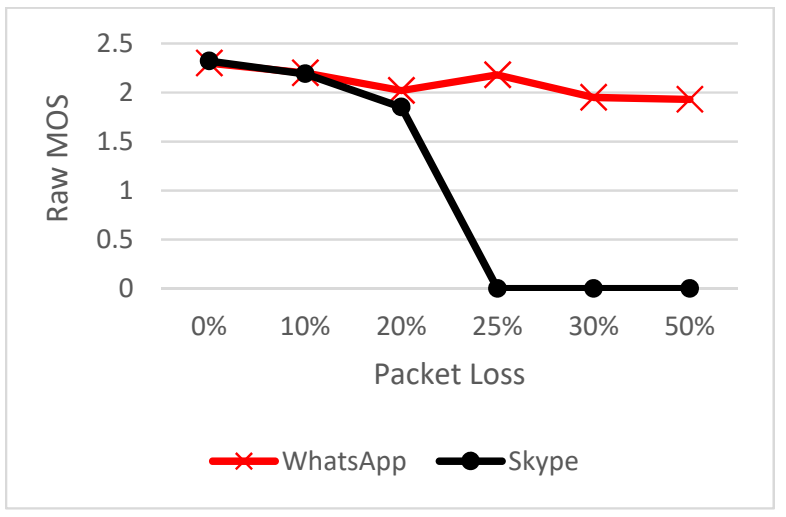

Fig. 6: Raw MOS vs packet loss of VoIP call

\section{CONCLUSIONS}

We have compared the performance of WhatsApp and Skype in terms of their data consumption when sending text messages and the quality of VoIP calls made with these two apps. Our results show that WhatsApp consumes less data compared to Skype in the sending of text messages and in the making of VoIP calls. We also find that WhatsApp is more resilient to packet losses compared to Skype, and its VoIP call quality is better as well. For a mobile phone user with a limited data plan, our results indicate that WhatsApp would be a more suitable application to use for sending text messages and making VoIP calls, compared to Skype.
For our future work, we plan to compare the performance of more instant messaging applications such as WeChat, Facebook Messenger, Line, and others. We also plan to investigate the performance of video calls when using these apps.

\section{REFERENCES}

[1] R. Dantas, C. Exton and A. Le Gear, "Comparing Network Performance of Mobile VoIP Solutions," 2018 6th IEEE International Conference on Mobile Cloud Computing, Services, and Engineering (MobileCloud), Bamberg, 2018, pp. 43-50.

[2] P. Wuttidittachotti, W. Akapan and T. Daengsi, "Comparison of VoIP-QoE from Skype, LINE, Tango and Viber over 3G networks in Thailand," 2015 Seventh International Conference on Ubiquitous and Future Networks, Sapporo, 2015, pp. 456-461.

[3] H. Xie and Y. Yang, "A Measurement-based Study of the Skype Peer-to-Peer VoIP Performance," The $6^{\text {th }}$ International Workshop on Peer-to-Peer Systems. IPTPS 2007, Feb 2007.

[4] C-N Chen, et al., "Measuring the Perceptual Quality of Skype Sources," in Proceedings of the 2012 ACM SIGCOMM workshop on Measurements up the stack (W-MUST '12).

[5] Zhang et al., "Modeling and Analysis of Skype Video Calls: Rate Control and Video Quality," IEEE Transactions on Multimedia, vol. 15 , no. 6,2013

[6] Exarchakos G., Druda L., Menkovski V. \& Liotta A., "Network Analysis on Skype End-to-End Video Quality," International Journal of Pervasive Computing and Communications, vol. 11, no. 1, 2015 pp. 17-42, 2015.

[7] T. Dargahi, A. Dehghantanha, M. Conti, "Forensics Analysis of Android Mobile VoIP Apps," Contemporary Digital Forensic Investigations of Cloud and Mobile Applications, Syngress, 2017

[8] C. Anglano, "Forensic Analysis of WhatsApp Messenger on Android Smartphones," Digital Investigation Journal, vol. 11, no. 3, pp.201-213, 2015.

[9] D. Bonfiglio, M. Mellia, M. Meo and D. Rossi, "Detailed Analysis of Skype Traffic," in IEEE Transactions on Multimedia, vol. 11, no. 1, pp. 117-127, Jan. 2009.

[10] Wireshark, available from https://www.wireshark.org/

[11] Clumsy, available from https://jagt.github.io/clumsy/

[12] PESQ tool, available from https://www.itu.int/rec/T-REC-P.862200102-I/en 